\title{
STRATIFIABLE SPACES AS SUBSPACES AND CONTINUOUS IMAGES OF $M_{1}$-SPACES
}

\author{
ROBERT W. HEATH AND HEIKKI J. K. JUNNILA ${ }^{1}$
}

\begin{abstract}
It is shown that every stratifiable space is the image of an $M_{1}$-space under a perfect retraction.
\end{abstract}

In 1961, J. Ceder introduced three classes of generalized metric spaces that he called $M_{i}$-spaces, $i=1,2,3$. Ceder showed that every $M_{1}$-space is an $M_{2}$-space and every $M_{2}$-space is an $M_{3}$-space, and he asked whether the converse results also hold [3]. C. Borges renamed $M_{3}$-spaces "stratifiable spaces" and proved several important results concerning these spaces in [1]. One half of Ceder's question was answered by G. Gruenhage and the second author of this paper, who showed, independently, that every stratifiable space is an $M_{2}$-space ([4] and [6]); the other half of the question has so far remained unanswered (for some partial answers, see [8] and [5]).

While the precise relationship between stratifiable spaces and $M_{1}$-spaces is yet to be determined, the following result sheds some light on that relationship.

TheOREM. Every stratifiable space is the image of an $M_{1}$-space under a perfect retraction.

Proof. Let $X$ be a stratifiable space. We assume that $X$ is a $T_{1}$-space; it is easy to modify the following proof so that it works also for non- $T_{1}$-spaces (note that stratifiable spaces are regular and hence "essentially $T_{1}$ ").

Denote by $S$ the subspace $\{0\} \cup\{1 / n \mid n \in N\}$ of the real line, and denote by $Z$ the space whose ground-set is the product-set $X \times S$ and whose topology is obtained by enlarging the product topology so as to make every point of the set $X \times\{1 / n \mid n \in N\}$ isolated.

It is not difficult to see that $Z$ is an $M_{1}$-space and that the projection $X \times S \rightarrow$ $X$ is a retraction from $Z$ onto $X$; however, to obtain a perfect retraction, we must construct a suitable subspace of $Z$. Since $X$ is an $M_{2}$-space, there exists a family $\mathscr{F}=\cup_{n \in N} \mathscr{F}_{n}$ of closed subsets of $X$ such that every point of $X$ has a neighborhood base consisting of members of $\mathscr{F}$ and, for every $n \in N$, the family $\mathscr{F}_{n}$ is closure-preserving. We may assume $X \in \mathscr{F}_{1}$. It follows from Theorems 3.14 and 4.8 of [6] that, for every $n \in N$, there exists a family $\mathcal{K}_{n}=\cup_{k \in N} \mathcal{K}_{n, k}$ of subsets of $X$ such that, for each $k \in N$, the family $\mathcal{K}_{n, k}$ is discrete, and, for each $x \in X$, there

Received by the editors April 25, 1980.

1980 Mathematics Subject Classification. Primary 54E20, 54C15, 54C25; Secondary 54C10, 54 B55.

${ }^{1}$ While working on this paper, the second author was visiting the University of Pittsburgh as an Andrew Mellon Postdoctoral Fellow. 
exists $K \in \mathscr{K}_{n}$ such that $x \in K \subset \cap\left\{F \in \cup_{m=1}^{n} \mathscr{F}_{m} \mid x \in F\right\}$. For each $K \in$ $\cup_{n \in N} \mathcal{K}_{n}$, let $x_{K}$ be an element of $K$. For every $n \in N$, let

$$
\mathcal{H}_{n}=\cup\left\{\mathcal{K}_{m, k} \mid m<n \text { and } k \leqslant n\right\},
$$

and let $A_{n}=\left\{x_{K} \mid K \in \mathcal{H}_{n}\right\}$; note that $\mathcal{H}_{n}$ is locally finite and, consequently, $A_{n}$ is discrete and closed. Let $Y$ be the subspace $(X \times\{0\}) \cup \cup_{n \in N}\left(A_{n} \times\{1 / n\}\right)$ of $Z$.

We show that $Y$ is an $M_{1}$-space. For all $F \in \mathscr{F}$ and $k \in N$, let

$$
F_{k}=\left[\left(\operatorname{Int}_{X} F\right) \times\{0\}\right] \cup[Y \cap(F \times\{1 / n \mid n>k\})] .
$$

We show that, for all $x \in X, F \in \mathcal{F}$ and $k \in N$, if $x \in F$, then ( $x$, $0) \in \mathrm{Cl}_{Y} F_{k}$. Let $F \in \mathcal{F}$ and $k \in N$, and let $x \in F$. To show that $(x, 0) \in \mathrm{Cl}_{Y} F_{k}$, assume on the contrary that $(x, 0) \notin \mathrm{Cl}_{Y} F_{k}$. Then there exists a neighborhood $G$ of $x$ in $X$ and $m \in N$ such that $(G \times\{1 / n \mid n \geqslant m\}) \cap F_{k}=\varnothing$. Let $E \in \mathcal{F}$ be such that $x \in E \subset G$, and let $h \in N$ be such that $\{E, F\} \subset \cup_{n=1}^{h} \mathscr{F}_{n}$. There exists $K \in \mathcal{K}_{h}$ such that $x \in K \subset E \cap F$. Let $i \in N$ be such that $K \in \mathcal{K}_{h, i}$, and let $j=h+i+k+m$. Then $\left(x_{K}, 1 / j\right) \in F_{k}$ and $\left(x_{K}, 1 / j\right) \in G \times\{1 / n \mid n>m\}$; this, however, is a contradiction since $(G \times\{1 / n \mid n \geqslant m\}) \cap F_{k}=\varnothing$. It follows that $(x, 0) \in \mathrm{Cl}_{Y} F_{k}$.

For every $n \in N$, let $\mathscr{B}_{n}=\left\{F_{k} \mid F \in \mathscr{F}_{n}\right.$ and $\left.k \in N\right\}$. We show that, for each $n \in N$, the family $\mathscr{B}_{n}$ is closure-preserving in $Y$. Let $n \in N$ and $\mathscr{B} \subset \mathscr{B}_{n}$, and let $y \in \mathrm{Cl}_{Y} \cup \mathfrak{B}$. If $y \in X \times\{1 / n \mid m \in N\}$, then $y$ is isolated and, consequently, $y \in \cup \mathscr{B}$. Assume that $y=(x, 0)$ for some $x \in X$. Let

$$
G=X \sim \cup\left\{F \in \mathscr{F}_{n} \mid x \notin F\right\}
$$

Since $\mathscr{F}_{n}$ is closure-preserving in $X$, the set $G$ is a neighborhood of $x$ in $X$, and hence the set $G^{\prime}=Y \cap(G \times S)$ is a neighborhood of $(x, 0)$ in $Y$. Since $(x, 0) \in$ $\mathrm{Cl}_{Y} \cup \mathscr{B}$, there exists $B \in \mathscr{B}$ such that $B \cap G^{\prime} \neq \varnothing$. Let $F \in \mathscr{F}_{n}$ and $k \in N$ be such that $B=F_{k}$. We have $F_{k} \cap G^{\prime} \neq \varnothing$ and consequently, $F \cap G \neq \varnothing$, that is $x \in F$. From the preceding part of the proof it follows that $(x, 0) \in \mathrm{Cl}_{Y} F_{k}$; in other words, that $y \in \mathrm{Cl}_{Y} B$. We have shown that the families $\mathscr{B}_{n}$ are closurepreserving in $Y$. Clearly, the family $\mathscr{B}_{0}=\{\{y\} \mid y \in Y(X \times\{1 / n \mid n \in N\})\}$ is $\sigma$-closure-preserving in $Y$. It is easily seen that the family $\cup_{n=0}^{\infty} \mathscr{B}_{n}$ is a base for the topology of $Y$. Since $X$ is a regular space, so is $Y$. Consequently, $Y$ is an $M_{1}$-space.

To complete the proof, denote by $f$ the restriction of the projection map $X \times S \rightarrow X$ to $Y$. It is easily seen that $f$ is a continuous mapping from $Y$ onto $X$ and that for each $x \in X$, the set $f^{-1}\{x\}$ is compact in $Y$. We show that the mapping $f$ is closed. Let $C$ be a closed subset of $Y$, and let $x \in X \sim f(C)$. Then $C \cap f^{-1}\{x\}=\varnothing$ and hence $(x, 0) \notin C$. Consequently, there exists a neighborhood $U$ of $x$ in $X$ and $m \in N$ such that $[U \times(\{0\} \cup\{1 / n \mid n>m\})] \cap C=\varnothing$. For each $n<m$, since $(x, 1 / n) \notin C$ and since $A_{n}$ is a closed and discrete subspace of $X$, the set $V_{n}=X \sim\left\{a \in A_{n} \mid(a, 1 / n) \in C\right\}$ is a neighborhood of $x$ in $X$. It is easily seen that the neighborhood $U \cap \cup_{n<m} V_{n}$ of $x$ is disjoint from the set $f(C)$. We have shown that the mapping $f$ is closed; hence $f$ is a perfect mapping. Setting $g(x)=(x, 0)$ for each $x \in X$, we obtain a homeomorphism between $X$ and the subspace $X^{\prime}=X \times\{0\}$ of $Y$. That $f$ is a retraction follows by observing that the restriction of the mapping $g \circ f$ to $X^{\prime}$ is the identity map on $X^{\prime}$. 
Since stratifiability is a hereditary property [3] and since the continuous image of a stratifiable space under a closed mapping is stratifiable [1], it follows from the above theorem that the problem, whether every stratifiable space is an $M_{1}$-space, is equivalent with some problems concerning the preservation of the $M_{1}$-property in topological operations.

COROLLARY. The following statements are mutually equivalent.

(i) Every stratifiable space is an $M_{1}$-space.

(ii) For every $M_{1}$-space, every closed subspace of the space is an $M_{1}$-space.

(iii) For every $M_{1}$-space, all the images of the space under perfect mappings are $M_{1}$-spaces.

The implication (ii) $\Rightarrow$ (iii) in the Corollary also follows from the result of $\mathbf{C}$. Borges and D. Lutzer that if every closed subspace of a space is an $M_{1}$-space, then every image of the space under a perfect mapping is an $M_{1}$-space [2].

Let us call a topological space an $M_{0}$-space if the topology of the space has a $\sigma$-closure-preserving base consisting of sets that are both open and closed. Every $M_{0}$-space is a strongly 0 -dimensional $M_{1}$-space. It is easily seen that a subspace of an $M_{0}$-space is again an $M_{0}$-space; from the result of Borges and Lutzer mentioned above it follows that the image of an $M_{0}$-space under a perfect mapping is an $M_{1}$-space. It is not known whether every $M_{1}$-space can be represented as the image of an $M_{0}$-space under a perfect mapping; for metrizable spaces, such a representation is obtained [7]. Note that it follows from the Theorem that if every $M_{1}$-space is the image of an $M_{0}$-space under a perfect mapping, then every stratifiable space is an $M_{1}$-space.

\section{REFERENCES}

1. C. J. R. Borges, Stratifiable spaces, Pacific J. Math. 17 (1966), 1-16.

2. C. J. R. Borges and D. J. Lutzer, Characterizations and mappings of $M_{1}$-spaces (R. F. Dickman and P. Fletcher, Eds., Topology Conf., Virginia Polytech. Inst. and State Univ., 1973), Lecture Notes in Math., vol. 375, Springer-Verlag, Berlin and New York, 1974, pp. 34-40.

3. J. Ceder, Some generalizations of metric spaces, Pacific J. Math. 11 (1961), 105-125.

4. G. Gruenhage, Stratifiable spaces are $M_{2}$, Topology Proc. 1 (1976), 221-226.

5.,$\sigma$-discrete stratifiable spaces are $M_{1}$ (preprint).

6. H. J. K. Junnila, Neighbornets, Pacific J. Math. 76 (1978), 83-108.

7. K. Morita, $A$ condition for the metrizability of a topological space and for n-dimensionality, Sci. Rep. Tokyo Kyoiku Daigaku, Sect. A 5 (1955), 33-36.

8. F. G. Slaughter, Jr., The closed image of a metrizable space is $M_{1}$, Proc. Amer. Math. Soc. 37 (1973), 309-314.

Department of Mathematics/Statistics, University of Pittsburgh, Pittsburgh, Pennsylvania 15260 